\title{
GaO (GNAO1) encephalopathies: plasma membrane vs. Golgi functions
}

\section{Gonzalo P. Solis and Vladimir L. Katanaev}

Heterotrimeric $G$ proteins are key signaling molecules best recognized as the immediate transducers of GPCRs (G protein coupled receptors). A heterotrimeric $\mathrm{G}$ protein complex consists of three subunits, $\alpha, \beta$, and $\gamma$, of which the $\mathrm{G} \alpha$ is responsible for binding to guanine nucleotides and to the cognate GPCR. Ligandactivated GPCRs catalyze the GDP-GTP exchange on $\mathrm{G} \alpha$ inducing the dissociation of $\mathrm{G} \alpha-\mathrm{GTP}$ from $\mathrm{G} \beta \gamma$, both being competent to engage downstream effectors. GTP hydrolysis returns the G $\alpha$ to its GDP-bound state allowing the formation of the heterotrimeric $\mathrm{G}$ protein, which binds the cognate GPCR for a subsequent activation cycle.

During the last five years, whole-exome sequencing of patients with severe infantile encephalopathies resulted in an avalanche of de novo heterozygous mutations in GNAO1 [1]. GNAO1 encodes for Gao, the major $\mathrm{G} \alpha$ subunit in the mammalian brain. Goo is one of the sixteen human $\alpha$-subunits and transduces the signal of a group of rhodopsin-like GPCRs including adrenergic, dopaminergic, opioid, and muscarinic receptor subtypes [2]. Gao knockout (KO) mice showed a strong developmental delay during the first 3 weeks after birth, multiple neurological abnormalities such as hyperalgesia, hyperactivity, generalized tremor with occasional seizures, and a severe impairment of motor control [2]. At the cellular level, dorsal root ganglion cells derived from $\mathrm{G} \alpha \mathrm{O}$ $\mathrm{KO}$ mice presented a reduced inhibition of $\mathrm{Ca}^{2+}$ channel currents by the activation of opioid receptors. Gao is additionally implicated in the regulation of $\mathrm{Ca}^{2+}$ and $\mathrm{K}^{+}$ channels in sensory and hippocampal neurons [2].

It has been proposed that Goo encephalopathies possess a strong genotype-phenotype association, with a group of mutations causing developmental delay and movement disorders, and another group responsible for the more severe cases of epileptic encephalopathy and Ohtahara syndrome [3]. Thus far, the vast majority of the cases is associated to single point mutations in 16 highly conserved residues [1], pointing to their involvement in basic Gao functions. The remaining two cases correspond to a deletion of seven amino acids and a predicted splicing defect [1]. A recent study indicates that mutations in eight of the 16 residues cause varying degrees of lossof-function downstream of the $\alpha 2 \mathrm{~A}$ adrenergic receptor [4]. Somehow contradictory, a previous study showed that two of these residues do not induce any significant defects compared to wild type Gao in the inhibition of $\mathrm{Ca}^{2+}$ currents by norepinephrine [5]. Intriguingly, mutations in other five residues did not compromise (or only slightly affected) $\alpha 2 \mathrm{~A}$ adrenergic receptor signaling [4]. Thus, it is highly plausible that Goo functions beyond its canonical role as GPCR-transducer also contribute to the development of Goo encephalopathies.

The functional localization of heterotrimeric $G$ proteins at the plasma membrane (PM) is widely accepted. However, an increasing amount of data indicate that they also work at different cellular compartments, especially at the Golgi apparatus [6]. Recently, we identified $>250$ proteins as potential Goo interacting partners, and subsequently uncovered a non-canonical Gao signaling involved in the regulation of vesicular trafficking at the Golgi [7]. We showed that two pools of Gao - at the PM and Golgi - coexist and act coordinately in cellular processes such as neuritogenesis: the PM Gao locally initiates neurite formation, and the Golgi Gao ensures

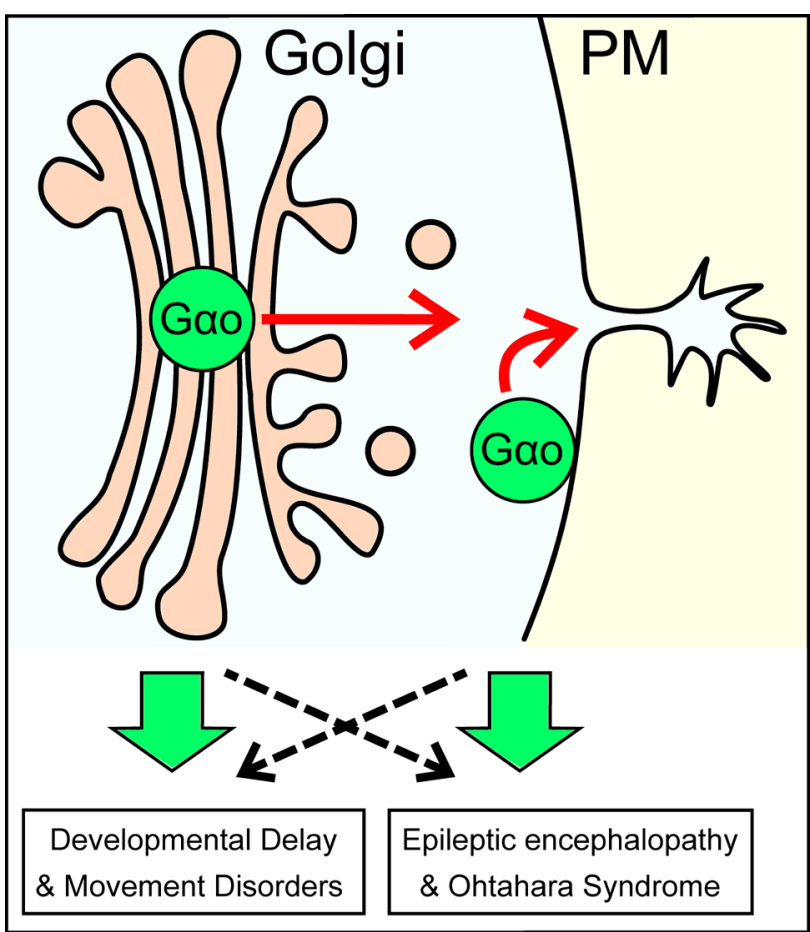

Figure 1: Goo mutations affecting differently its PM and Golgi functions as the potential origin of the varying degrees of severity observed in GNAOI encephalopathies. 
material delivery for neurites to grow (Figure 1). We nailed down a novel mechanism of unconventional interactions at the Golgi, where an atypical GPCR (KDEL receptor) stimulates G $\beta \gamma$-free Goo, which in turn activates the small GTPases Rab1 and Rab3. This pathway is highly conserved from fly to human, and is also required for synaptogenesis in vivo [7].

Remarkably, mutations in the Goo-coupled $\alpha 2 \mathrm{~B}$ adrenergic receptor are linked to a familial epilepsy (ADRA2B, OMIM:607876 [8]), and several de novo mutations in the heterotrimeric $\mathrm{G} \beta 1$ are associated to severe neurodevelopmental disorders often accompanied by seizures (GNB1, OMIM:616973). On the other hand, some of the Golgi partners of Gao [7] have been involved in diverse neuropathies characterized by developmental delay and intellectual disability but not seizures (i.e. COG6, OMIM:615328; AP1S1, OMIM:609313; GDI1, OMIM:300849). Therefore, it is tempting to speculate that different degrees of severity in GNAOI encephalopathies reflect the extent in which the PM vs. Golgi functions of Gao are affected by a particular mutation (Figure 1). We hypothesize that mutations targeting mainly the canonical GPCR-mediated signaling of Gao induce the most severe cases of epileptic encephalopathy, whereas mutants affecting predominantly its Golgi role develop neurodevelopmental delay, intellectual disability and movement disorders.

To conclude, the next challenge to eventually complete our understanding of the etiology of GNAOI encephalopathies is the generation of a tractable animal model to study the Gao pathways that control development and physiology of the central nervous system. Such a model should allow for large screening of chemicals to identify hit compounds in order to develop personalized drugs against $\mathrm{G} \alpha$ o encephalopathies.

Vladimir L. Katanaev: Department of Pharmacology and Toxicology, University of Lausanne, CH-1011 Lausanne, Switzerland; School of Biomedicine, Far Eastern Federal University, Vladivostok, Russian Federation

Correspondence to: Vladimir L. Katanaev, email vladimir. katanaev@unil.ch

Keywords: heterotrimeric GaO, GNAOl encephalopathy, GPCR, Golgi apparatus

Received: September 07, 2017

Epub: October 25, 2017

Published: May 08, 2018

\section{REFERENCES}

1. Schorling DC, et al. Neuropediatrics. 2017; 48:371-377. https://doi.org/10.1055/s-0037-1603977.

2. Jiang M, et al. Neurosignals. 2009; 17:23-41. https://doi.org/10.1159/000186688.

3. Menke LA, et al. J Child Neurol. 2016; 31:1598-601. https://doi.org/10.1177/0883073816666474.

4. Feng H, et al. Neurology. 2017; 89:762-70. https://doi.org/10.1212/WNL.0000000000004262.

5. Nakamura K, et al. Am J Hum Genet. 2013; 93:496-505. https://doi.org/10.1016/j.ajhg.2013.07.014.

6. Luini A, et al. Curr Opin Cell Biol. 2016; 39:37-42. https://doi.org/10.1016/j.ceb.2016.01.014.

7. Solis GP, et al. Cell. 2017; 170:939-55 e24 https://doi.org/10.1016/j.cell.2017.07.015.

8. Amberger JS, et al. Nucleic Acids Res. 2015; 43:D789-98. https://doi.org/10.1093/nar/gku1205.

Copyright: Solis et al. This is an open-access article distributed under the terms of the Creative Commons Attribution License 3.0 (CC BY 3.0), which permits unrestricted use, distribution, and reproduction in any medium, provided the original author and source are credited. 